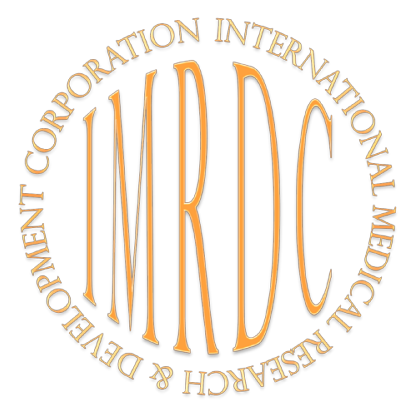

\title{
Cardiology
}

\section{Regulatory Synchronization of Hemodynamics of the Heart and Brain in Norm}

\author{
Alexander G. Kruglov, PhD, ScD*; Valery N. Utkin; Alexander Yu. Vasilyev, PhD, ScD; \\ Andrey A. Kruglov, PhD \\ Central Research Institute of Radiation Diagnosis \\ Moscow, the Russian Federation
}

\begin{abstract}
By catheterization, the integral indicators of synchronization and interaction of blood flows, designated as "venous and arterial boluses," were obtained, studied and analyzed in healthy people on the pathway: right heart-lung-left heart. It has been confirmed that the complete CC of the BB from RA to the ejection from the LV has a length equal to two completed heart contraction cycles. Interaction of venous and arterial boluses, with differentiated external myocardial exposure, along the path "venous block of the heart-lung-arterial block of the heart," forms averaged (compensated by the flexible septum) variable pressure values between the stages of intracardiac routes of BBs (unidirectional, synchronous, but spread in the space). The complex of these pressure values creates an intracardiac pressure balance at the border of high- and low-energy processes of the heart. We defined the sequential dynamics of these values as CMIP. Our mathematical and graphical data demonstrate the presence of direct and inversecardio-cerebral wave connections, where the waveguides are the vessels of entry and exit from the skull. We believe that CMIP is a universal, central rhythmic process, a regulator that determines the sequence and intensity of the CC phases, HR, and synchronous nervous and wave effects on brain structures. The modulating effect of CMIP on brain structures, providing some sensory-motor reactions, behavioral functions and forms of behavior, occurs outside the realm of consciousness. Our data suggest that the modulating effect of CMIP on the brain is carried out not only along the neural pathways, but also by the vascular wave structures that combine the heart and brain into a single hydrodynamic structure with phase-varying volume and configuration, as well as variable patterns of regulatory impulses. (International Journal of Biomedicine. 2019;9(4):281-286.)
\end{abstract}

Key Words: cardiac cycle $\bullet$ hemodynamic parameters $\bullet$ cardio-cerebral synchronization $\bullet$ ECG

\section{Abbreviations}

Ao, aorta; AV, aortic valve; ANS, autonomic nervous system; BB, "bolus" of the blood; BP, blood pressure; CaS, carotid siphon; CC, cardiac cycle; CS, coronary sinus; CMIP, cardiac mean integral pressure; DP, diastolic pressure; EF, ejection fraction; HR, heart rate; IJV, internal jugular vein; IVS, interventricular septum; $\mathbf{L V}$, left ventricle; $\mathbf{M V}$, mitral valve; PV, pulmonary valve; PPW, peripheral pulse wave; PW, pulse wave; PWV, pulse wave velocity; RV, right ventricle; RA, right atrium; RHV, right hepatic vein; SC, systemic circulation; SS, sigmoid sinus; SV, stroke volume; TV, tricuspid valve.

\section{Basic Part}

The purpose of this study was to determine the mechanisms of CMIP formation and its interaction with brain structures.

In previous articles, ${ }^{(1-9)}$ we have given the hemodynamic

*Corresponding author: Alexander G. Kruglov, PhD, ScD. Central Research Institute of Radiation Diagnosis. Moscow, the Russian Federation.E-mail: krag48@mail.ru indices obtained by catheterization in various vascular areas, as well as the relationship between parameters of the cerebral, central and peripheral blood flow analyzed by methods of mathematical statistics. The results we present (Table 1) were obtained by synchronization of ECG, PPW, CMIP and SS data. The SS curve, in contrast to the previous ones (constructed by average values), is a real curve obtained during the examination of a 43-year-old male patient, the combined results of which made it possible to exclude the suspected diagnosis. The 
results were obtained with the subject in the supine position (in a position perpendicular to the vector of gravity when its influence in all parts of the venous system is equal) and on exhalation (elimination of the effect of changes in intrathoracic pressure). The hemodynamic and metabolic parameters obtained by catheterization through a probe that was wedged in the upper bulb of IJV are the wave and metabolic parameters of the sigmoid venous sinus of the brain (SS).

The information thesaurus of the SS venous blood flow does not contain extracranial blood admixture but contains, along with other parameters, hemodynamic parameters and metabolites of blood flows from the cavernous sinus (including the dynamics of $\mathrm{CaS}$ impulses), transverse sinus, and straight sinus (vein collector from deep brain structures), and pressor (wave) effect of the dynamics of the pial chamber. Note that for fast processes that exceed the inertial characteristics of the outgoing flows, the cranial cavity is considered as a sealed formation.

We showed the presence of direct synchronized hemodynamic cardio-cerebral connections in previous ${ }^{(1,3,6-8)}$ articles. It was established that the active processes in LV and Ao (systolic and pulse pressure) have no relationship to SS pulse pressure, providing perfusion dynamics of the brain starting from the Ao mean pressure. SS pressure levels are linked (Table 1) ${ }^{(1)}$ with the LV ejection period, the achievement of maximum pressure in the LV-Ao system at the opening of $\mathrm{AV}$, and the formation of a maximum pressure at the vascular entrances into the cranial cavity. As is known, LV systole creates a pulse dissipative wave structure in the aortic trunk, reaching all points of the body before the closing of $\mathrm{AV} .^{(10)}$ We believe that the negative correlation between the pressure levels in SS, LV and Ao is explained by the damping effect of CaSs (located in the cavernous sinus), which change the internal lumen under the influence of the incoming Ao wave impulse.

$\mathrm{CaSs}$ are conductors of the wave structures of the heart (informational characteristics of different levels of control) interfering with the cavernous venous sinus of the brain. In norm, the changes in the internal lumen of CaSs eliminate pressure values, which exceed the Ao mean pressure, providing optimal levels of perfusion pressure in the brain.. The excess pressor effect of intravascular pressure is utilized by the ligamentous apparatus of CaSs and transfer pressor effect on the venous blood of the cavernous sinus (connected through the sinus petrosus superior and inferior with SS), which we recorded at the exit from the venous system of the brain as the SS wave synchronous with "X"- collapse of the RA (i.e., LV systole) (Table Norm). ${ }^{(6)}$ In other words, this is the energetic, active phase, during which the synchronized wave impulses of LV and intracranial venous pressure have a unidirectional vector opposite to the vector of the venous blood flow dynamics (IJV-RA).

Perfusion dynamics of the brain is provided starting of the Ao mean pressure level (Table Norm) ${ }^{(1)}$, positively conjugated to SS from the DP level, which is an indicator of the formation of a single hydrodynamic system in the phase of diastolic evolution (from the medium to diastolic pressure): Ao-vessels entering the skull-SS-IJV-RA (Table Norm ${ }^{(1)}$ ). This explains the positive conjugation of the diastolic evolution of the intracranial venous bed with aortic diastolic evolution. Achieving the minimum values of the Ao-SS-RA gradient is replaced by LV systole. During one CC, the relationship AoSS pressure goes through a linking cycle: "-", "0", “+”.

Thus, the fixation point of the parameters of intracranial venous blood flow (SS), which includes the information wave resources from the actual LV systole (CASs and pial chamber) and venous outflow from the brain, is a stage of a single hemodynamic pathway (without valves all along the way from the metabolic fields of the brain to the tricuspid valve), regulated by wave impulses of vessels of the inflow and outflow of the brain. We gave a preliminary analysis of the formation and interaction of BBs (IC-1, IC-2 and CMIP) in previous works. ${ }^{(6-9)}$

Minimal CMIP, influencing the formation of the RV spheroid, determines the pressure level of the "trigger point," which forms the level of the PV valve opening pressure and RVEF into the lung system, where there is a final transformation (hemodynamic and metabolic) of the "venous bolus" of the right heart into an "arterial bolus" passing into the left parts of the heart. Maximal CMIP determines the pressurized level of the opening of AV and LVEF going to the exchange zones. The indicated sequentially synchronized stage of CC (Ao-RV) constitutes a pathway, which a pulse wave impulse passes: LV-Ao-CASs, pial chamber-SS-SJV-RA-RV (with opened TV and closed MV).

Thus, during one phase of CC (LV contraction), the pulse wave passes through the hemodynamic pathway: LVRV. CMIP is simultaneously 1) an integral derivative of the total interaction between all hemodynamic paths (incoming, transit and outgoing) and the structural elements of the heart; and 2) a derivative of the pressor factor of the myocardium, which regulates the conditions of formation, interaction, synchronization, and vectors of passage with BBs of the right and left parts of the heart of the corresponding tracts.

We believe that the primary link in the sequence of intracardiac transformations of BBs is the pressor effect of functional syncytium, involving the cascade dynamics of the synchronous interaction of different zones of the myocardium, valve, and fibrous and ligamentous apparatus of the heart, creating an extended synchronized pressor-depressive effect of the myocardium as a whole on the contents of the heart chambers during $\mathrm{CC}$. With the trigger type of response, syncytium has synchronized differences in the developed power and the sequence of contraction in different areas of the myocardium. The result is differences in the initial pressor parameters for the formation of BBs, giving them inertia and a vector. Boluses 1 and 2, simultaneously and unidirectionally passing intracardiac tracts, have differences in the power of the pressor effect of the myocardium of the venous and arterial parts of the heart (the value that forms the «bolus» of pressure), as well as other systematic coordinate differences (density, viscosity, gas composition, electromagnetic properties, etc.). Boluses 1 and 2 interact through anatomically separated formations with different plasticity properties: a section of the upper RA wall (which is not an interatrial septum) and the ascending part of Ao; RA and LA through the oval window and atrial septum; RA with LV through the membranous 
portion of IVS; RV with LV through IVS and others. As a result, throughout $\mathrm{CC}$ in the zones of contact of BBs on the path "venous block of the heart-lung-arterial block of the heart," averaged (compensated by plastic septa) variable pressure values are formed between synchronous, but spatially separated stages of intracardiac traces "venous and arterial boluses" (between the right and left chambers of the heart). Their combination creates an intracardiac pressure balance that determines the state of "current equilibrium" on the border between high- and low-energy processes of the heart (right and left parts), the sequential dynamics of which are indicated on the graph: CMIP, in combination with BBs. ${ }^{(9)}$

We believe that at the borders and in the interaction zones, the combined pressor and other parameters of BBs, with the external influence of the myocardium, form a single regulatory structure that balances the intracardiac pressor imbalance. This structure is CMIP-a systemic, intracardiac hemodynamic regulator, which is an integral function of myocardial dynamics and BBs. We believe that the echocardiographic methods, visualized the amplitude fluctuations of the intracardiac formations, reflect fragments of the structure that forms the CMIP. In other words, the phase architectonics of the vibrating flexible, structural heart formations forms the intracardiac structure of a variable configuration, which is the physical basis for the formation of integral CMIP.

In our opinion, CMIP is a nonlinear, multidimensional regulatory structure of the heart as a whole, which distributes and differentiates in intensity and duration components (pressor, electrical, magnetic, rheological, and other), the changes of which inevitably cause a cascade of changes in the subsystems. CMIP, as a system regulator of a higher regulatory level, the first in a hierarchy of regulators, exerts a dominant regulatory influence on parameters (pressure, speed, vector, etc.), ensuring the synchronism of the dynamics of Boluses 1 and 2.

The critical threshold value of the change in CMIP parameters - derived from the dynamics of the myocardium as a whole and the whole set of qualities of the hemodynamic flows of the heart, including the total information thesaurus of the large and small circles of the systemic blood flowrequires a significantly larger number of changes in the input parameters to change systemic stability than each bolus separately; systemic stability is an integral indicator (and regulator) of the next lower level, the second, in the hierarchy of systemic regulatory levels (Boluses 1 and 2). In other words, CMIP, as a system, has greater stability than each component controlled by it, determining their parametric stability and intrasystem balance. The intensity of the effect of the CC phases on the modulation of the activity of brain structures, due to the afferentation level from mechanoreceptors and baroreceptors of the heart and blood vessels, ${ }^{(11)}$ in our opinion, also depends on the direct action of the LV-Ao wave pulses (information patterns consisting of frequency and intensity parameters) on brain structure. We consider direct evidence of this relationship are the results of our correlation analysis and graphical indicators of the ratio of CMIP and SS (Table 1), which coincide in the main peak values and profiles of CMIP (integral indicator of cardiac activity) and SS, the information thesaurus of which includes the final hemodynamic and metabolic indicators of brain metabolism. Organ hemodynamics (the third regulatory level), the blood flows of which are combined into RA, transforms into a single hemodynamic formation a venous blood bolus, the total thesaurus of which includes the entire total information volume (hemodynamic and metabolic) of SC. We consider it necessary to note that in addition to metabolites, all flows entering RAs include hormones from the endocrine glands (IJV from the pituitary and thyroid glands; IVC from the adrenal glands, genital organs, etc.; RHV in transit through the liver from the pancreas, etc.), where they are mixed with myocardial metabolites from the actual systole (CS), structuring the venous bolus.

The combination of metabolites, gas functional ${ }^{(4)}$ and hormones from the exchange zones is an informational result of the current state of homeostasis, changing in accordance with the needs of the mechanisms of homeostatic adaptation (i.e., status and needs of homeostasis). In this paper, we do not consider in detail the mechanisms of intracardiac transformation of BBs. BB, undergoing the transformation of the complete $\mathrm{CC}$ - the right heart ("venous bolus"), the lung exchange field (including the thesaurus of the pulmonary circulation), the left heart ("arterial bolus") - is a formed structure of the second regulatory level and goes during the LV systole into the vascular bed of the exchange fields, as the managing homeostatic complex (wave and metabolic). We believe that differences in the interpretation of the scatter of results obtained when determining SV $(55 \mathrm{ml}-90 \mathrm{ml})$ and LVEF $(50 \%-80 \%)^{(12)}$ are explained by a homeostatically adaptive contractile function of the heart. In other words, each myocardial contraction has its own initial regulatory mechanism: an integral variable 3rd regulatory level (current status of the homeostasis) that forms the venous bolus of RA. The set of its parameters forms the first regulatory level (CMIP) of the heart as a whole, which determines ("calibrates") the variation in the composition and volume of the second regulatory level (BBs of both circles of blood circulation) adequate to homeostatic needs, which goes to the exchange fields of large and small circles of blood flow. CMIP, composing an integral derivative of hemodynamics of the large and small circles of blood flow with the dynamics of constructive formations of the heart, and being the first hierarchical regulatory level, affects the thirdorder regulators by the second-order regulator: LV systolic BB (EF) and pulse wave variations. Thus is formed a closed cycle of direct and reverse wave relationships of the first through third hierarchical levels of the regulatory central (cardiac) and peripheral (organ) controls of the wave patterns of the body as a whole.

The idea of a central rhythmic process that determines the heart rhythm and synchronous behavioral (and electrophysiological) equivalents was advanced by Velden and Juris in 1975. ${ }^{(13)} \mathrm{A}$ distinction was made between the systolic and diastolic evoked potentials on the $\mathrm{EEG}^{(14,15)}$ with the difference in the leads for the hemispheres of the brain and differences in synchronization with the pulse wave of the carotid artery. Many studies have shown the dependence 
of behavioral reactions and EEG on HR, as well as the dependence of the sensorimotor reaction time on the CC phase, ${ }^{(15)}$ including in patients with an artificial pacemaker. ${ }^{(16)}$ The possibility of a direct effect of heart pulsations on brain tissue was emphasized, ${ }^{(15)}$ the direct connection of which, through the influence of $\mathrm{CC}$ on intracranial venous pressure, is shown in Table 1. With a direct effect on brain tissue, in our opinion, rheological blood parameters are very significant (density and viscosity - functions of volume and fluidity), in the absence of the receptor apparatus, which we assigned to the primarily regulated functions of the homeostatic control. ${ }^{(1)}$ Significant inhibition of cortical activity in the systole phase with hypertension was noted, with a decrease in the level of perception and cognitive abilities, ${ }^{(15)}$ which Sandman et al. associated with "bar-receptor inhibition." At the same time, it was found that rapid responses of cerebral vessels to auditory signals were accompanied, depending on the $\mathrm{CC}$ phase, by changes in the intracranial blood volume-in response to stimuli given during the diastole. ${ }^{(17)}$

Many studies ${ }^{(18,19)}$ have found changes in the cortical potentials of EEG caused by heart contraction under the influence of a conscious change in the vector of voluntary attention and additional motivation. In other words, cardiocerebral synchronous processes, the initiator of which is $\mathrm{CC}$, are registered. The interpretation of these relationships is based on ideas about the phase effect on cortical activity from baroreceptors and mechanoreceptors of the heart and blood vessels. The totality of our data, both mathematical and graphic, demonstrating the coincidence and mutual influence of the hemodynamic parameters of the heart and brain, allows us to supplement the concept of "adaptive modulation of the activity of mental functions with afferentation from the cardiovascular system" ${ }^{(11,20-22)}$ with the fact of the presence of direct and reverse regulatory hemodynamic connections of the heart and brain. We consider the totality of these connections to be an autonomous (partially duplicating nervous regulation), direct, and feedback-wave regulatory channel. As an additional argument in favor of the double (nervous and wave) effect of $\mathrm{CC}$ on the modulation of brain structures (sensory and motor reactions), we consider changes in the motor response of the heart to an external impulse with a denervated heart, ${ }^{(23)}$ as well as a change in the motor response of a transplanted (denervated) human heart to physical activity: increase (twice) in cardiac output and SV $(>40 \%)$. Motor changes in the reaction of a denervated heart, devoid of afferent and efferent connections with the brain, in our opinion, confirm the presence of two partially independent, complementary pathways of the regulatory interaction of the heart and brain: hemodynamic and nervous channels. These changes indicate a deficiency of regulatory functions in combination: 1) autonomous cardiac and 2) wave (hemodynamic) regulatory mechanisms, in the absence of 3) central nervous influence, under the conditions of additional external stimuli.

In a previous publication, ${ }^{(9)}$ we presented our ideas about the mechanism of autonomous regulation of $\mathrm{CC}$ through the pressor effect of intracardiac hemodynamics on the sinoatrial node. ANS, which provides sensory afferentation from the heart and blood vessels, has a low speed of nerve impulses (the thinnest myelinated fibers of A-type, B-type and nonmyelinated C-fibers): from $0.5 \mathrm{~m} / \mathrm{s}$ to $18 \mathrm{~m} / \mathrm{s}$. PWV, which in contrast to the linear blood flow velocity increases with distance from the pulse generator (heart), is from $4 \mathrm{~m} / \mathrm{s}$ to 12 $\mathrm{m} / \mathrm{s}{ }^{(24)}$ Thus, the rate of afferentation of nerve impulses from the baro- and mechanoreceptors of the heart and blood vessels along the ANS trunks is comparable to the speed of wave hemodynamic impulses, PW from the heart to the structures of the brain (and other organs). In other words, we establish the presence of two (duplicating and mutually complementary) ways of regulatory influence on the brain structures initiated by $\mathrm{CC}$ through the nervous and wave (hemodynamic) structures.

We believe that the data we have provided allow us to supplement and expand the semantic, theoretical, and practical content of Lacey's theory with new arguments. In particular, an increase in HR and BP, leading to an increase in afference from arterial baroreceptors, prevents the receipt of information from the environment into the projection zones of the cortex of the brain, thereby contributing to the optimization of cognitive processes.

Reduced baroreceptor inhibition (decreased HR and BP) leads to improved perception of external signals ("intake"). We attach particular importance to the synchronization of the $\mathrm{CC}$ phases and the alpha rhythm of the brain detected in depressive syndromes, ${ }^{(15)}$ as well as their synchronization in the cerebral cortex of the occipital zones, which have no confirmed connection with the regulation of cardiac activity. ${ }^{(25,26)}$ The aforementioned testifies, in our opinion, to a much larger, in comparison with existing views, range of influence of $\mathrm{CC}$ on the activity of the brain and mental derivatives.

We believe that a targeted study of the synchronization of the CC phases with variations in the rhythmic processes of brain activity (magnetograms, alpha rhythm and other waves) across the entire spectrum of mental norm and pathology is promising. The relevance of this vector of research is confirmed by the results of many works, in particular, the synchronization of the alpha rhythm with the CC phases, the differentiation of the phases and the CC rhythm in affective disorders, vital and neurotic depressions, ${ }^{(13,15,27)}$ accounting for $30 \%-57 \%$ of visits to cardiologists, accompanied by sensations (often treated as senestopathies ) in the projection zones of the heart, subjectively formulated as "pain, heaviness in the soul." It should be noted that other emotional statesemotional uplift, enthusiasm and many others, formulated as "connected with the soul" - are subjectively (both normal and pathological) also felt in these projection zones.

\section{Conclusion}

Thus, CMIP (multidimensional integral indicator of pressor dynamics of the myocardium during a complete CC) is a universal central rhythmic process, a regulator of the first hierarchical level, determining the sequence and intensity of the $\mathrm{CC}$ phases, HR and synchronous nervous and wave effects on brain structures. The action of this systemic regulator occurs outside the realm of consciousness, exerting a modulating effect on brain structures that provide some sensory-motor reactions, behavioral functions and conscious forms of behavior. 


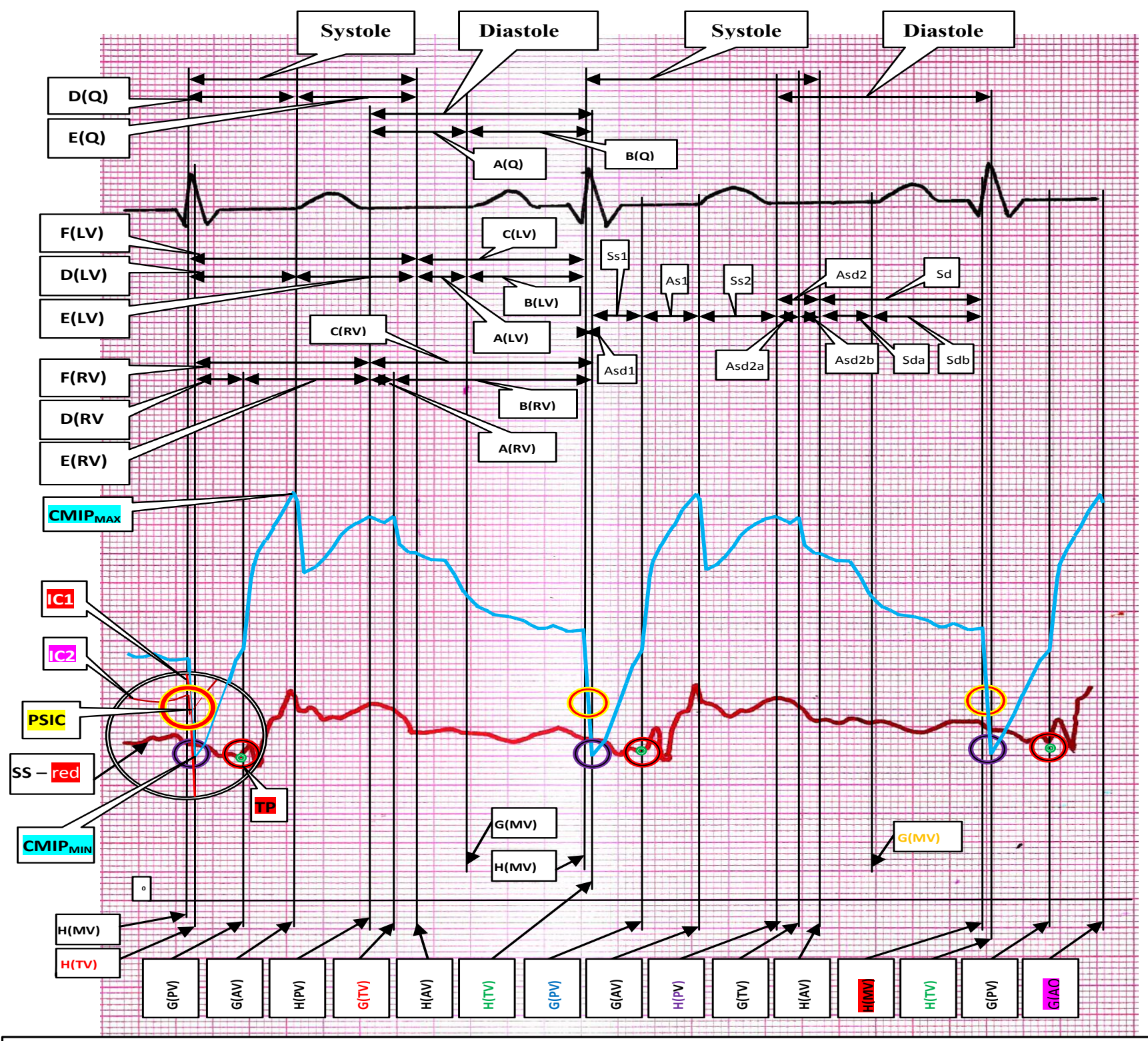

\begin{tabular}{|c|c|}
\hline $\begin{array}{l}\text { A(Q) - isometric ventricular relaxation } \\
\text { B(Q) - actual ventricular diastole } \\
\text { C(LV) - LV diastole } \\
\text { A(LV) - isometric LV relaxation } \\
\text { B(LV) - actual LV diastole } \\
\text { C(RV) - RV diastole } \\
\text { A(RV) - isometric RV relaxation } \\
\text { B(RV) - actual RV diastole } \\
\text { D(Q) - isometric ventricular contraction } \\
\text { E(Q) - actual ventricular systole } \\
\text { F(LV) - LV systole } \\
\text { D(LV) - isometric LV contraction } \\
\text { E(LV) - actual LV systole } \\
\text { F(RV) - RV systole } \\
\text { D(RV) - isometric RV contraction } \\
\text { E(RV) - actual RV systole } \\
\text { G(AV) - opening of AV } \\
\text { H(PV) - closing of PV } \\
\text { G(TV) - opening of TV } \\
\text { H(AV) - closing of AV }\end{array}$ & $\begin{array}{l}\text { G(MV) - opening of MV } \\
\text { H(TV) - closing of TV } \\
\text { H(MV) - closing of MV } \\
\text { G(PV) - opening of PV } \\
\text { H(PV) - closing of PV } \\
\text { Acd1 - asynchronous period of ventricular systole-diastole -1 } \\
\text { Ss1 - synchronization period of isometric ventricular contraction -1 } \\
\text { As1 - asynchronous period of ventricular systole -1 } \\
\text { Ss2 - synchronization of the actual ventricular systole -2 } \\
\text { Asd2 - asynchronous period of ventricular systole-diastole -2 } \\
\text { Asd2a -from closing of PV to opening of TV } \\
\text { Asd26- from opening of TV to closing of AV } \\
\text { Sd - period of synchronization of ventricular relaxation } \\
\text { Sda - isometric relaxation of LV } \\
\text { Sdb - actual LV diastole } \\
\bar{O} \text { - integral curves (IC-1 and IC-2) } \\
\text { - TP including ZTEP: SS-VH-SVC-CS-RV-LA } \\
\text { - intersection point for ZTEP } \\
\text { - point of "stabilization" of ICs of the right and left parts of the heart } \\
- \text { CMIP }\end{array}$ \\
\hline
\end{tabular}


The modulating effect on brain structures that provide sensory-motor reactions and behavioral functions is carried out through two mutually complementary (duplicate) paths of synchronous regulation: 1) the neural tract-baroreceptors, mechanoreceptors of the heart and vascular bed, and 2) the vascular tract-wave hemodynamic structures having propagation velocities comparable to the nerve impulse. Waveguides are the vessels of entry and exit from the skull, providing direct and reverse control connections of the heart and brain, combining the heart and brain into a single hydrodynamic system with phase-changing volume, configuration and variable patterns of regulatory impulses.

\section{Competing Interests} interests.

The authors declare that they have no competing

\section{References}

1. Kruglov AG, Gebel GYa, Vasilyev AY. Impact of IntraExtracranial Hemodynamics on Cerebral Ischemia by Arterial Hypertension (Part 1-2). Int J Biomed. 2012;2(2):96-101.

2. Kruglov AG, Vasilyev AY, Sherman VA. Human dynamic homeostasis control matrix in the norm with psychophysiological aspects. New York: IMRDC; 2016.

3. Gebel GYa, Dasaev AN, Belichenko IA, Kruglov AG, Gudenko VV, Utkin VN. [Intracranial venous pressure in the norm and arterial hypertension]. Abstracts of the 7th Union Conference on Space and aerospace medicine. Kaluga;1982:184. [Abstract in Russian].

4. Kruglov AG, Utkin VN, Vasilyev AY. The Role of Integrated Gas Compounds in Regulation of Gas Homeostasis in the Norm. International Journal of Biomedicine. 2017; 7(3):185-191.

5. Kruglov AG, Utkin VN, Vasilyev AY, Sherman VA. Human Homeostatic Control Matrix in Norm. International Journal of Biomedicine. 2016;6(3):184-9.

6. Kruglov AG, Utkin VN, Vasilyev AY. Synchronization of Wave Flows of Arterial and Venous Blood with Phases of the Cardiac Cycle in Norm: Part 1. International Journal of Biomedicine. 2018;8(2):123-128.

7. Kruglov AG, Utkin VN, Vasilyev AY, Kruglov AA. Synchronization of Wave Flows of Arterial and Venous Blood and Phases of the Cardiac Cycle with the Structure of the Peripheral Pulse Wave in Norm: Part 2. International Journal of Biomedicine. 2018;8(3):177-181.

8. Kruglov AG, Utkin VN, Vasilyev AY, Kruglov AA. Synchronization of Wave Flows of Arterial and Venous Blood and Phases of the Cardiac Cycle with the Structure of the Peripheral Pulse Wave in Norm: Part 3. International Journal of Biomedicine. 2018;8(4):288-291.

9. Kruglov AG, Utkin VN, Vasilyev AY, Kruglov AA. Synchronization of Wave Flows of Arterial and Venous Blood and Phases of the Cardiac Cycle. (Part 4). International Journal of Biomedicine. 2019;9(2):106-110.
10. Lightfoot A. Transport phenomena in live systems. Biomedical aspects of momentum and mass transport. M.: Mir; 1977. [in Russian].

11. Lacey J.I. Somatic response pattering and stress: some revisions of activation theory. In: Appley MH, Trumbull R, editors. Psychological stress: Issue in research. New York: Appleton-Century-Croft; 1967:14-42.

12. Malov YuS, Yarovenko II. [Significance of the left ventricular ejection fraction in diagnosis of heart failure]. Vestnik Rossiiskoi Voenno-Medicinskoi Akademii. 2018;1(61):68-74. [Article in Russian].

13. Velden M, Juris M. Perceptial perfomans as a function of intra-cycle cardial activity. Psychophysiology. 1975;12(6):685-92.

14. Sandman CA. Augmentation of the auditory event related potentials of the brain during diastole. Int J Psychophysiol. 1984;2(2):111-9.

15. Sandman CA, Walker BB, Berka C. Influence of afferent cardiovascular feedback on behavior and the cortical evoked potential. In: Cacioppo JT, Petty RE, edittors. Perspectives in cardiovascular psychophysiology. Guilford, New York; 1982:189-222.

16. Callaway E 3rd, Layne RS. INTERACTION BETWEEN THE VISUAL EVOKED RESPONSE AND TWO SPONTANEOUS BIOLOGICAL RHYTHMS: THE EEG ALPHA CYCLE AND THE CARDIAC AROUSAL CYCLE. Ann N Y Acad Sci. 1964;112:421-31.

17. Sandman CA, O'Halloran JP, Isenhart R. Is there an evoked vascular response? Science. 1984;224(4655):1355-7.

18. Weitkunat R, Cestaro V, Katkin E. Evidence for a lateralized heartbeat evoked potential. Psichophysiology. 1989;26:65.

19. Weitkunat R, Schandry R. Motivation and heartbeat evoked potentials. J. Psychophysiol. 1990;4:33-40.

20. Lacey JI, Lacey BC. Studies of heart rate and other bodily processes in sensorimotor behavior. In Obrist $\mathrm{P}$, Black AH, Brener J, DiCara LV, editors. Cardiovascular psychophysiology. Chicago: Aldine; 1974:538-564.

21. Lacey JI, Lacey BC. Some autonomic-central nervous system interrelationships. In Black P, editor. Physiological correlates of emotion. New York: Academic Press; 1970:205-228.

22. Lacey JI, Lacey BC. On heart rate response and behavior: a reply to Elliot. J Pers Soc Psychol. 1974;30(1):1-18.

23. Donald DE, Shepherd JT. Sustained capacity for exercise in dogs after complete cardiac denervation. Am J Cardiol. 1964;14:853-9.

24. Pokrovsky VM, Korotko GF. Human physiology. M. Meditsina; 2003. [In Russian].

25. Walker BB, Walker JM. Phase relations between carotid pressure and ongoing electrocortical activity. Int $\mathrm{J}$ Psychophysiol. 1983;(1):65-73.

26. Walker BB, Sandman CA. Visual evoked potentials change as heart rate and carotid pressure change. Psychophysiology. 1982;19(5):520-7.

27. Have B, Britton B, Daniels D, Heilman K, Porges $\mathrm{S}$, Halaris A. Low cardiac vagaltone index by heart rate variability differentiates bipolar from major depression. World J Biol Psychiatry. 2019;20(5):359-367. doi: 10.1080/15622975.2017.1376113. 